\title{
Femtosecond laser writing of near-surface waveguides for refractive-index sensing
}

\author{
Alain Abou Khalil, ${ }^{1,2,}{ }^{*}$ Philippe Lalanne, ${ }^{3}$ (D) Jean-Philippe \\ Bérubé, ${ }^{1}$ Yannick Petit, ${ }^{2,4}$ Réal Vallée, ${ }^{1}$ and Lionel \\ CANIONI ${ }^{2}$

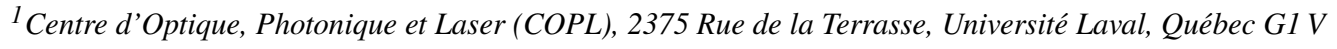 \\ A06, Canada \\ ${ }^{2}$ Université de Bordeaux, CNRS, CEA, CELIA, UMR 5107, F-33405 Talence, France \\ ${ }^{3}$ Laboratoire Photonique, Numérique et Nanosciences (LP2N), IOGS, Université de Bordeaux, CNRS, \\ 33400 Talence cedex, France \\ ${ }^{4}$ Université de Bordeaux, CNRS, ICMCB, UPR 9048, F-33608 Pessac, France \\ *alain.abou-khalil@u-bordeaux.fr
}

\begin{abstract}
Femtosecond laser writing of optical waveguides and components in glasses has been a remarkably growing research field during the last two decades. However, such laserinscribed optical components were mostly written within the volume of the glass due to the unavoidable ablation that arises when the focal spot is approaching the glass surface. This has generally limited the interaction of light with the surrounding medium thus preventing sensing functionality. In this paper, we present the inscription of surface and near-surface silver based waveguides in a silver containing glass with no need for additional processing as it is the case for standard type I waveguides. In addition, an ultra-sensitive refractive index sensor in a $1 \mathrm{~cm}$ glass chip is obtained based on near-surface waveguides interacting with liquid droplets acting as top-layer on the glass surface. Remarkably, the device exhibits a novel double-wing feature that sharpens the response and enhances its sensitivity. Our results highlight the advantages of silver based waveguides paving the way towards further surface based sensors in fibers.
\end{abstract}

(C) 2019 Optical Society of America under the terms of the OSA Open Access Publishing Agreement

\section{Introduction}

In recent years, the use of fiber optics for sensing applications has gathered a growing interest. Optical sensing provides in-situ, fast, selective, remote, real time and non-destructive sensing. It is generally based on the interaction of light with the outer medium. The fiber sensors are generally based on the evanescent wave interaction with the outer medium. Many evanescent wave based fiber sensors were proposed during the past years for gas detection [1], chemical detection [2,3] and refractive index sensing [4-6]. However, to manufacture such sensors, the fiber has to be tapered or a part of its cladding must be removed so as to allow the evanescent wave to overlap with the sensing medium. In 1987, a segment of a step index multimode fiber was heated and expanded for methane detecting applications based on the evanescent wave interaction [1]. Another sensor was designed where the plastic cladding of a silica core fiber was removed to detect nitrite compounds in water [3]. Also, refractive index (RI) optical sensors were manufactured in fibers comprising Bragg gratings. The shift in the reflected Bragg wavelength was monitored as a function of the refractive index changes [7-9]. On the other hand, Polynkin et al. demonstrated a RI sensor where an SMF 28 fiber was tapered so as to allow its evanescent field to carry an important fraction of the guided power [10]. The optical transmission was monitored as a function of the different refractive index liquids. A similar work was reported but in a plastic fiber where a segment of the plastic cladding was removed to create the sensing medium [5]. In this report, a theoretical model was proposed based on Fresnel's equation that describes reflection and transmission of the guided light inside the fiber [5]. In fact, almost all 
reported RI sensors to date required etching or removing of a part of the fiber cladding in order to functionalize the fiber and create the sensing medium.

Finally, one should distinguish between most chemical sensors which are based on the absorption of the evanescent wave and refractive index sensors which rely on the phase change resulting from the variation of the surrounding medium refractive index affecting the spatial mode confinement.

Femtosecond laser writing (fs LW) technique is a well-established technique allowing to directly inscribe three-dimensional (3D) structures inside transparent materials. More specifically, waveguides [11-17] and optical components [18] were embedded inside glasses using fs laser writing, yet only a few studies were reported on laser writing waveguides neighboring the glass surface [19-22]. This is due mainly to the glass ablation that occurs when focusing near the surface. The interest of writing near-surface/shallow optical waveguides is to allow the interaction of light with the outer medium in order to create sensors in glass chips and optical fibers. Still, few works were reported for the interaction of shallow waveguides with the surrounding medium [21-25], knowing that writing near-surface waveguides is not easily achievable due to the decrease of the pulse energy threshold for glass modification when approaching the glass surface [26]. For instance, Lapointe et al. managed to make a RI sensor by reproducing the same idea reported by Bilro et al. [5], i.e. writing a near surface waveguide interacting with the outside medium in a reinforced Gorilla bulk glass while monitoring the transmission power as a function of the different RI liquids deposited on the near-surface portion of the waveguide [19]. Yet, the reported near-surface waveguides were still up to $5 \mu \mathrm{m}$ away from the surface. Bérubé et al. overcame this limitation by an additional process based on placing a cover glass slide in optical contact with the top surface of the glass [20]. This allows the suppression of the air-glass interface allowing the creation of the nearest waveguides to the glass surface compared to previously reported works [21-25].

In the case of the silver containing glasses used in this work, fs LW process induces the formation of silver clusters on the periphery of the interaction voxel that are accountable for a refractive index change $(\Delta \mathrm{n})$, called type Argentum (type $A$ ) as reported in our previous papers [27-29]. One can say that "Type $A$ " is a smooth modification in the low pulse energy regime where the migration and creation of silver clusters occurs. This process allows for the creation of silver based optical waveguides called type $A$ waveguides [28]. In this paper, we take advantage of type A modification allowing for the direct inscription of surface and near-surface waveguides with no need for additional processing or compensation method. To illustrate the potential of this process, an ultra-sensitive RI sensor was demonstrated based on type A near-surface waveguides that exhibits a double-wing feature in the response of the waveguide transmission as a function of the RIs enabling an extended sensing range. Finally, a theoretical model was developed to precisely account for light propagation within the near-surface waveguides by properly handling the spatial mode modification related to the varying refractive index of the surrounding medium. This model is showing a remarkably good agreement with the experimental results, especially in terms of describing the observed double-wing feature of our sensor.

\section{Experimental method}

\subsection{Glass samples}

The glass sample used was a silver containing zinc phosphate glasses manufactured by Argolight (AG01 ArgoGlass). The sample dimensions are $10 \mathrm{~mm}$ x $10 \mathrm{~mm}$ x $900 \mu \mathrm{m}$. The facets were cut and polished to optical quality prior to the writing process in order to optimize light injection as well as visualization of the waveguides and the guided mode profiles. 


\subsection{Femtosecond laser writing}

The glass samples were irradiated using a femtosecond Yb-doped laser oscillator (Clark Impulse), $360 \mathrm{fs}$ pulse duration (FWHM) at $12.6 \mathrm{MHz}$ and emitting at $1030 \mathrm{~nm}$ wavelength. The sample displacement and positioning was carried out using a high precision 3D translation stages (Aerotech, ANT 130 and planar DL - 200XY).

The near-surface waveguides were written using linear polarization, laser irradiance of 7.2 $\mathrm{TW} / \mathrm{cm}^{2}$ and a writing speed of $50 \mu \mathrm{m} / \mathrm{s}$ corresponding respectively to a pulse energy of $28 \mathrm{~nJ}$ and $4 \times 10^{5}$ pulses. A $100 \mathrm{X}-0,8$ NA Nikon microscope objective was used to focus the laser pulses inside the glass sample.

\subsection{Mode profile characterization setup}

Near field mode profiles were observed after injecting light from a $630 \mathrm{~nm}$ diode laser using a single mode fiber (HP-460 with core diameter $2.5 \mu \mathrm{m}$ - NA = 0.13) into the waveguides. Then, a 100X - 0.55 NA Mitutoyo objective was focused on the output facet to visualize the guided mode profile and conjugate it onto a CCD camera. A beam splitter was installed before the CCD camera to split the light between the CCD camera and a power meter to monitor the transmission drop following the oil deposition on the glass surface.

\subsection{Top-layer refractive index characterization}

Cargille matching index fluids were used (hereafter designed as oil) with refractive index ranging from 1.4 to 1.632. A micropipette was used to deposit $3 \mu \mathrm{L}$ droplets on the glass surface in a controlled way. The dimensions of the droplets were monitored and controlled using a top view camera and a $1.25 \mathrm{X}$ microscope objective. Following every oil droplet deposition, the glass surface was cleaned using Ethanol until the initial transmission was recovered before the next oil deposition.

\subsection{Refractive index change measurement}

The refractive index change $(\Delta \mathrm{n})$ between the pristine glass and the waveguides was measured using a phase-contrast microscopy method with commercially available wave-front sensor SID4Bio from PHASICS Inc [28]. A 100X - 1.3 NA oil immersion objective was used to image the structures using white light illumination.

\section{Results and discussion}

\subsection{Near-surface waveguides}

Femtosecond Laser Writing (fs LW) of near-surface waveguides was performed inside silvercontaining zinc phosphate glass. Following fs LW in such silver containing glasses, fluorescent silver clusters are generated at the vicinity of the interaction voxel based on the non-linear multi photon absorption process [30,31]. The reader is referred to Refs. [30-34] for the details on the process of creation of silver clusters in silver containing glasses. In summary, the writing process could be explained as a laser quill inducing the creation of silver clusters on the periphery of the interaction voxel while the high laser intensity photo-dissociate the silver clusters created in the middle due to the Gaussian beam profile [31,34]. Those silver clusters are responsible for a refractive index change allowing the fabrication of optical waveguides and components [28].

Following a stair pattern, series of $1 \mathrm{~cm}$ waveguides were written at different depths from the glass surface as shown in Fig. 1(a). The S-shaped waveguides were in fact comprising segments of approximately $5 \mathrm{~mm}$ neighboring the surface and thus acting as the sensing region (Fig. 1(b)). The conditions of laser writing were optimized in order to avoid ablation when the focal point approached the glass surface. 
(a)

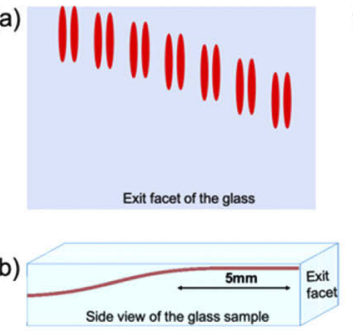

(c)

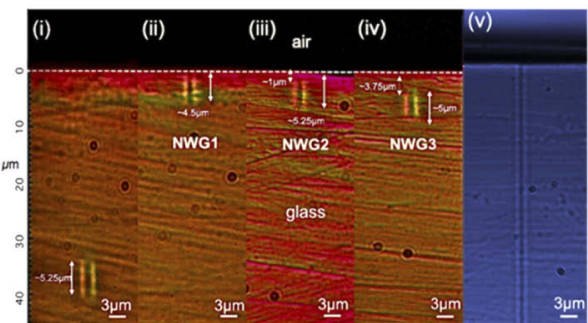

Fig. 1. Schematic presentation of (a) the stair pattern fs LW process (b) side view of the sample following fs LW of a rising near-surface waveguide. (c) Exit facet bright field images for (i) well embedded waveguide inscribed $32 \mu \mathrm{m}$ below the surface (ii) NWG1, waveguide written on the glass surface (iii) NWG2, waveguide $1 \mu \mathrm{m}$ below the glass surface (iv) NWG3, waveguide $3.75 \mu \mathrm{m}$ below the glass surface (v) Top view of NWG2 near-surface waveguide portion. No ablations nor cracks were observed indicating a smooth writing process.

Figure 1(c) shows the exit glass facet under white light illumination of a straight waveguide written $\sim 32 \mu \mathrm{m}$ below the glass surface (acting as a reference) as well as three near-surface waveguides, NWG1, NWG2 \& NWG3. One can see that the writing process results in smooth modification whereas no ablations or mini explosions were observed from both, side and top view of the near-surface waveguides (Fig. 1(c)). Moreover, the waveguides' morphology of double parallel planes (typical of type A morphology [28,32]) as well as the dimensions $(5.25 \mu \mathrm{m}$ $\mathrm{x} 3 \mu \mathrm{m})$ are well maintained even when the waveguides are neighboring the glass surface. One notes however that the waveguide NWG1 (Fig. 1(c.ii)) is showing a smaller transverse length as compared to the three others ( $4.5 \mu \mathrm{m}$ as compared to $5.25 \mu \mathrm{m})$. This is due to the fact the writing process occurred on the glass surface and a part of the waveguide is cropped by the glass - air interface. Even though the writing occurred directly on the glass surface, still no crack nor ablation were observed for this waveguide.

To test the waveguiding and sensing properties of such waveguides, a $630 \mathrm{~nm}$ laser was coupled inside the waveguides (see Experimental methods section). A single mode aspect was observed for all the written waveguides. For the NWG1 waveguide, the mode profile was observed to be different from a typical type A single mode profile well buried inside the glass surface [28]. Moreover, for the waveguides embedded inside the glass, their spatial mode profiles were not the same as a function of their distance from the surface. Waveguides that are less than $\sim 2.5$ $\mu \mathrm{m}$ from the surface (e.g. NWG2), exhibited some distortion of their near-field mode profile as shown in Fig. 2(a). The guided modes in that case are very likely still affected by the glass-air interface. However, compared to guided modes for waveguides that are deeper inside the glass ( $>3 \mu \mathrm{m}$ ), the mode profiles were not affected as shown in Fig. 2(b) for NWG3. In that case, the spatial mode profiles are identical to the one observed at $160 \mu \mathrm{m}$ below the surface reported in our earlier work [28].

The refractive index change $(\Delta \mathrm{n})$ between the pristine glass and the written waveguides was measured using the wave front sensor (see Experimental methods section) for all the written waveguides. A moderate $\Delta \mathrm{n}$ of $2 \times 10^{-3}$ was measured for the portion of waveguides neighboring the glass surface which is comparable to the measured $\Delta \mathrm{n}$ for a single mode waveguide well buried inside the glass $\left(\Delta \mathrm{n}=2.5 \times 10^{-3}\right.$ for a waveguide written $160 \mu \mathrm{m}$ below the glass surface) as previously reported [28]. As expected, two positive $\Delta \mathrm{n}$ peaks were observed which is typical of type A modification [27,28] as shown in Fig. 3. Note that the pulse energy threshold for glass modification decreases when approaching the glass surface [26], which complicates the inscription of near-surface waveguides, especially with a relatively high $\Delta$ n, i.e. larger than $1 \times 10^{-3}$. In comparison, near-surface waveguides with a relatively low $\Delta \mathrm{n}$ of $5 \times 10^{-4}$ were 

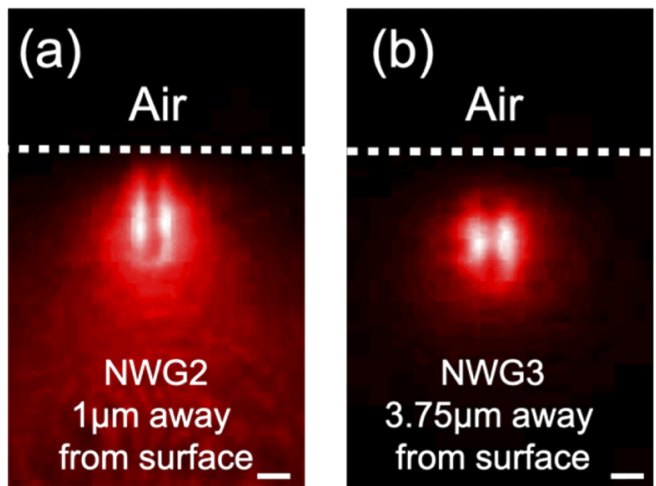

Fig. 2. Experimental near-field mode profiles for (a) NWG2 and (b) NWG3 waveguides following the injection of a $630 \mathrm{~nm}$ laser. The scale bar is equal to $2 \mu \mathrm{m}$. The spatial mode profile of the waveguide NWG2, $1 \mu \mathrm{m}$ away from the surface, is affected by the glass-air interface whereas NWG3's mode ( $3.75 \mu \mathrm{m}$ away from the surface) is not.

reported by Lapointe et a lin a reinforced gorilla glass [17] while a moderate $\Delta \mathrm{n}$ of $2.9 \times 10^{-3}$ was reported by Bérubé et al in borosilicate glass using a compensation method [20].
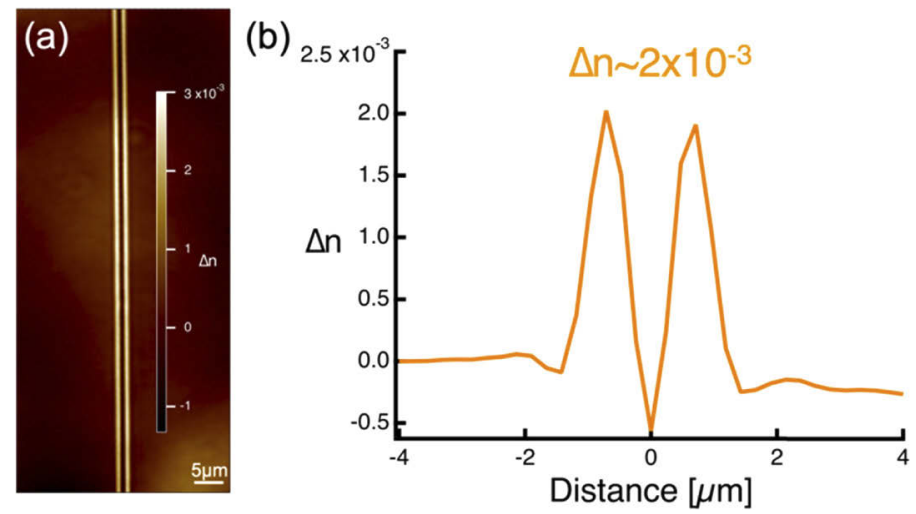

Fig. 3. (a) Top view phase image of the rising portion of a near-surface waveguide (b) Refractive index change $(\Delta \mathrm{n})$ profile extracted from the phase image in Fig. 3(a). A moderate $\Delta \mathrm{n}$ value of $2 \times 10^{-3}$ was measured for the portion of the waveguide neighboring the glass surface.

The sensitivity of all the written waveguides following a stair pattern (Fig. 1(a)) were tested by injecting a $630 \mathrm{~nm}$ laser and dropping a $4 \mathrm{~mm}$ droplet of Cargille oil exhibiting a refractive index (RI) of 1.604 (matching the RI of the waveguide) on the near-surface portion of the waveguide. The oil was dropped using a micropipette in order to control and maintain the same droplet size for all the measurements (see Experimental methods section). The optical transmission drop was monitored following the deposition of the oil droplet on the glass surface for different waveguides as a function of their distance from the surface, as shown in (Fig. 4). The transmission for each waveguide is normalized with respect to the transmission of the waveguide when no oil was deposited on the sample $T_{M} / T_{1}\left(n_{o i l}=1\right)$. The distance from the surface is defined by the distance from the glass surface to the top of the waveguide as shown in (Fig. 1(c)).

Even though the NWG1 waveguide was written on the surface, it does not exhibit the highest sensitivity. This is due to the fact that the guided mode is heavily cropped at the glass-air interface. Therefore, the waveguide's measured transmission is affected by experimental uncertainty due 


\section{Optics EXPRESS}

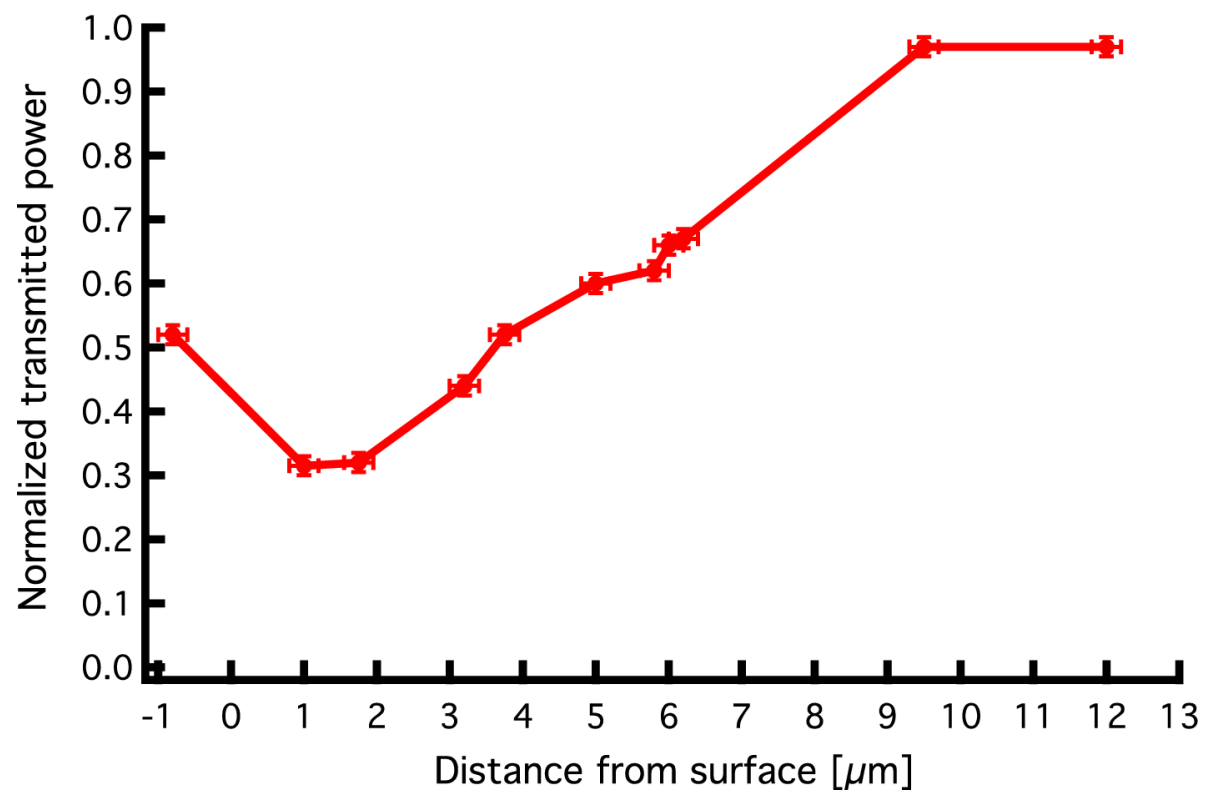

Fig. 4. Normalized transmission power of the written near-surface waveguides as a function of their distance from the surface following the deposition of $4 \mathrm{~mm}$ oil droplet with a refractive index matching that of the glass $\left(\mathrm{n}_{\mathrm{oil}}=1.604\right)$.

to the lossy nature of the waveguide. The highest sensitivity was shown for waveguides 1 to 2 $\mu \mathrm{m}$ below the surface with a transmission drop of $\sim 65 \%$ (4.5dB) for a $4 \mathrm{~mm}$ interaction length with the oil droplet. As expected, the sensitivity of the waveguides decreases as they go deeper inside the glass. Yet, even the deepest waveguide (at $7 \mu \mathrm{m}$ from the glass surface) interacting with the oil droplet exhibits a significant transmission drop of $\sim 33 \%(1.73 \mathrm{~dB})$ highlighting the high sensitivity of such waveguides.

Compared to the of work by Lapointe et al. where the transmission drop is $\sim 15 \%(0.7 \mathrm{~dB})$ for a $1 \mathrm{~cm}$ long interacting region [19], our waveguides seems to be much more sensitive. We believe that is impossible to write type I waveguides neighboring the surface $(\sim 1 \mu \mathrm{m}$ below the surface without additional processing i.e. reinforced glass or compensation method) as it is can be achieved using type $A$ waveguides. This is due to the fact that the type A occurs before type $I$ in the energy pulse scale, by triggering the silver photochemistry without affecting the glass matrix structure [27]. The fs LW process targets the chemicals inside the glass i.e. silver ions and induces the creation of silver clusters. The high sensitivity of the here reported waveguides compared to type I waveguides reported in [19], is probably correlated to their remarkable proximity to the glass surface $(\sim 1 \mu \mathrm{m})$. One should mention, that the propagation losses of the presented waveguides is estimated to exhibit upper bounds of $1.2 \mathrm{~dB} / \mathrm{cm}$ using the estimation method reported in [28].

\subsection{Refractive index sensor}

A refractive index (RI) sensor was developed based on the near-surface waveguides reported herein. Two waveguides NWG2 and NWG3 were considered, featuring distances from the surface of 1 and $3.75 \mu \mathrm{m}$ respectively. Different index matching oils were deposited and covered over 4 $\mathrm{mm}$ on top of the near-surface portions of the waveguides. Then, the transmission was monitored for each waveguide as a function of the oil RI. The results are depicted in Fig. 5. Surprisingly, NWG2 exhibited an unexpected double-wing feature characterized first by a transmission increase 


\section{Optics EXPRESS}

up to $\sim 50 \%$ for an oil RI of 1.602 but followed by a steep decrease for an oil RI of 1.604 $\left(n_{\text {oil }} \sim n_{\text {eff }}\right)$ (see Visualization 1). The transmission decrease was expected from previous reports for similar sensors [4,5,19]. As for the transmission increase observed for $n_{\text {oil }}<n_{\text {eff }}$, it was observed by Digonnet et al [35] in the context of side-polished fiber couplers, and by Polynkin et al in the context of RI sensing application [4].

(a)

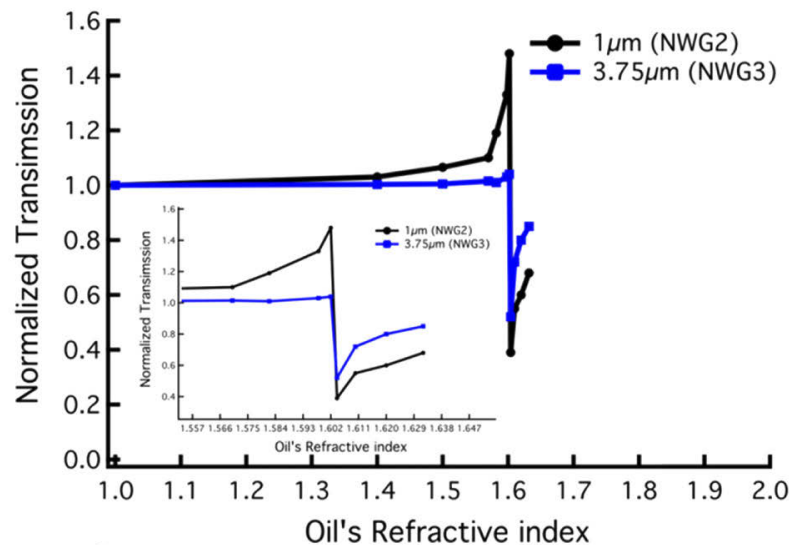

(b)

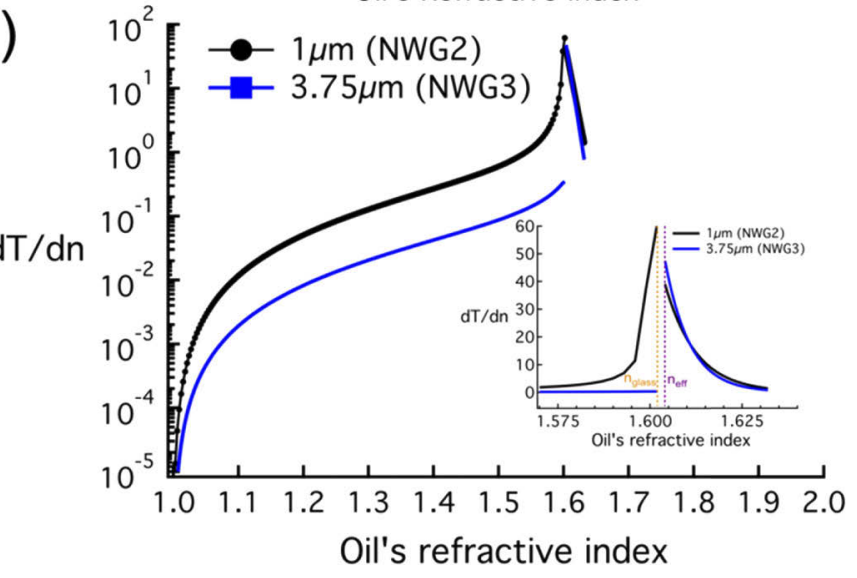

Fig. 5. (a) Normalized transmission of experimental values following the deposition of $4 \mathrm{~mm}$ oil droplets exhibiting different refractive indices for a near-surface waveguide (black) $1 \mu \mathrm{m}$ away from the surface (NWG2), (blue) $3.75 \mu \mathrm{m}$ away from the surface (NWG3). NWG2 exhibits a non-standard original response with a two wing-feature. Inset: Close-up of the double-wing feature (see Visualization 1). (b) The sensing sensitivity corresponding to the derivative of the normalized transmission as a function of the refractive index (dT/dn) presented in logarithmic scale for: NWG2 (black) and NWG3 (blue) in the different sensing regions. Inset: Close-up of the sensitivity $(\mathrm{dT} / \mathrm{dn})$ in the double-wing feature zone in linear scale (1.57 to 1.632$)$.

In the case of our sensor, NWG2 exhibited more losses (i.e. $\mathrm{T}_{1}$ reduced accordingly for $n_{\text {oil }}=1$ ) as compared to deeper waveguides although the waveguides were all written using the same writing parameters. As soon as the RI of the oil matches that of the glass, the glass-air interface disappears and so does the mode distortion/mismatch so that the waveguiding conditions are restored as for the case of the waveguide deeply embedded. That explains the losses recovery and the corresponding increase of the relative transmission. One should note that not all the 
losses were recovered knowing that the oil droplet is only $4 \mathrm{~mm}$ compared to a $5 \mathrm{~mm}$ waveguide portion neighboring the surface.

The double-wing feature was much less pronounced for the more deeply embedded NWG3 waveguide, as shown in (Fig. 5 - blue curve). The main feature in that case is the decrease of the transmission observed for oil's RI matching with the waveguide's RI or more precisely the effective mode RI ( $\left.n_{\text {oil }} \sim n_{\text {eff }} \sim 1.604\right)$ as observed in the previous reported works $[4,5,19]$. The sensing behavior could therefore be described here in terms of three different regions. The first region (region I) where the oil's RI is lower or equal to the glass's, $n_{\text {oil }} \leq n_{\text {glass }}=1.602$, second region (region II) where the oil's RI is equal to the propagating effective mode RI $n_{\text {oil }} \sim n_{\text {eff }}$ and the third (region III) is where the oil's RI is higher than the effective mode RI $n_{\text {oil }}>n_{\text {eff }}$. In the first region $\left(n_{\text {oil }} \leq n_{\text {glass }}\right)$ the light is guided inside the waveguide by total internal reflection (TIR) with a slight augmentation of the transmission (2 - 4\%). It could be correlated to a lower Fresnel reflection coefficient between the propagating mode and the interface following the deposition of oil droplets with higher RI compared to air $(\mathrm{n}=1)$. The second region is where $n_{\text {oil }} \sim n_{\text {eff }} \sim 1.604$, a large portion of light is ejected outside the waveguide core knowing that the deposited oil matches the RI of the waveguide's core. Finally, the third region $n_{\text {oil }}>n_{\text {eff }}$ is where the light is guided in the waveguide based on a leaky mode exhibiting some losses while propagating in the waveguide.

In Fig. 5(b), we have calculated the normalized transmission derivative as a function of the refractive index (dT/dn) which defines the sensitivity of our device (a direct estimation from experimental data of Fig. 5(a)). One can see that such sensitivity is not constant and varies with respect to the three RI regions. For NWG2 exhibiting the double-wing feature: region I ( $n_{\text {oil }} \leq n_{\text {glass }}$ ) the sensitivity increases progressively to reach its peak in this region at $n_{\text {oil }} \sim n_{\text {glass }} \sim 1.604$ where we observe an increase of the transmission of $\sim 50 \%$ (Fig. 5(a) black curve). Then, in region II (between $n_{\text {glass }}$ and $n_{\text {eff }}$ ) a very large sensitivity is observed (value of about -550 , not shown in Fig. 5(b)) due to mode leaking out of the waveguide (sudden transmission drop). Finally, for region III, $n_{\text {oil }}>n_{\text {eff }}$ the sensitivity decreases as the RI increases. So, for NWG2, the device exhibits different responses for three possible sensing regions while exhibiting the highest response in region II $\left(n_{\text {oil }} \sim n_{\text {eff }}\right)$. For NWG3: the slight increase in the response in region $\mathrm{I}$ is almost not detected and no sensing could occur in that zone. In that case, only two sensing regions are possible, $n_{\text {oil }} \sim n_{\text {eff }}$ and $n_{\text {oil }}>n_{\text {eff }}$. Therefore, this kind of surface waveguides provide a response comparable to that of the previously reported RI sensors [5,19]. The inset in Fig. 5(b), shows a close-up of the sensitivity in linear scale in the range of 1.57 to 1.632 highlighting the importance of the waveguide proximity to the glass surface to achieve different sensing regions. One should mention that it is noticeable that the double-wing feature (for NWG2) allows a non-null sensitivity for oil indices below the glass index, even for indices down to the 1.33-1.37 range compatible with biological and environmental applications, which cannot be achieved with the previously reported RI sensors $[5,19]$.

Finally, we report in Table 1 a sensitivity comparison taking into consideration the interaction/sensing zone, the transmission drop magnitude and range between our RI sensor and the previously reported conceptually-similar sensors is presented in Table 1. One can see, that the herein reported sensor is the most sensitive using fs laser writing with a transmission drop of $82 \%$ for an interaction zone of $5 \mathrm{~mm} \times 3 \mu \mathrm{m}$ compared to a $15 \%$ transmission drop for a $10 \mathrm{~mm}$ x $11 \mu \mathrm{m}$ interaction zone for Lapointe et al. [17,19] . Moreover, our sensor exhibits a comparable sensitivity with fiber-based sensors as shown in Table 1. Furthermore, one should not forget that it is based in a $1 \mathrm{~cm}$ glass chip which is probably the most compact device compared to the previously reported RI sensors based on the same concept idea [4,5,19]. 
Table 1. Dimensions of the interaction zone, magnitude and refractive index range of the transmission drop for different refractive index sensors previously reported and our sensor.

\begin{tabular}{lllll}
\hline Authors & Technique & Interaction zone & Transmission drop & RI range for transmission drop \\
\hline Polynkin et al [4] & Fiber optic based & $10 \mathrm{~mm} \times 140 \mu \mathrm{m}$ & $100 \%$ & $1.402-1.459$ \\
Bilro et al [5] & Fiber optic based & $19.35 \mathrm{~mm} \times 650 \mu \mathrm{m}$ & $80 \%$ & $1.417-1.492$ \\
Lapointe et al [17,19] & Fs laser writing & $10 \mathrm{~mm} \times 11 \mu \mathrm{m}$ & $15 \%$ & $1.5114-1.5117$ \\
Current work & Fs laser writing & $5 \mathrm{~mm} \times 3 \mu \mathrm{m}$ & $82 \%$ & $1.602-1.604$ \\
\hline
\end{tabular}

\subsection{Theoretical model}

In contrast to previous theoretical models devised to study the behavior of such refractive index sensors that are based on Fresnel equations and total internal reflection of light [5,19], we propose a new modeling approach based on the full Maxwell equations solution for the propagation of electromagnetic field. The model focuses on the spatial mode profile propagating inside the embedded waveguide and the near-surface waveguide as a function of different oil's RI ( $1 \rightarrow 1.75)$.

Generally, the transmitted power in waveguides is carried out by guided modes (presently a single mode is used) and a large number of radiation modes which decay due the power leakage in the cladding. Possible excitation of radiation modes arises from the mode mismatch during the light coupling process. However, in our analysis, the radiation modes in the embedded part of the waveguide are already damped before reaching the near-surface waveguide. Therefore, the typical type A single mode is injected as the initial condition to solve the Maxwell equations in the near-surface waveguide.

The theoretical analysis is performed using an in-house aperiodic Fourier-Modal method (a-FMM) [36]. The latter is a fully-vectorial method that solves Maxwell's equations by expanding the radiation and guided modes in Fourier series in the transverse directions of the waveguide by using a scattering-matrix formalism in the propagation direction. The method was initially elaborated to study high-index-contrast photonic-crystal waveguides [37] and has been successfully used afterwards to analyse a variety of problems in plasmonics and photonics. [38]. It is analytical in the propagation direction, which represents an advantage compared to brute-force full-discretization methods for analyzing optical structures with mm-long waveguide sections. The waveguide was simulated as two rectangular shapes spaced by $1.75 \mu \mathrm{m}$, exhibiting $5 \mu \mathrm{m}$ length and $500 \mathrm{~nm}$ thickness with a step refractive index change of $\sim 2 \times 10^{-3}$ placed $1 \mu \mathrm{m}$ away from the glass surface (Fig. 6(b)) which reflects the experimental values and conditions.

The geometry considered in the theoretical analysis is depicted in the inset of Fig. 6(a). In a first step, we consider all possible modes, the guided and radiations one, in the near-surface waveguide portion interacting with the oil, called "oil section" hereafter, and compute the transmission $T_{t}=|t|^{2}$ of the fundamental mode of the "air section". Then, we consider the transmission $T_{F P}$ predicted with a Fabry-Perot (FP) model, for which only a single mode (either guided for $n_{\text {oil }}<n_{\text {eff }}$ or leaky for $n_{\text {oil }}>n_{\text {eff }}$ ) of the oil section is assumed to carry the power transmission. In the numerical implementation, the mode is selected by computing and selecting the largest coupling coefficient $T_{M}$ at the air - oil waveguide interface between the incident guided mode of the air section (inset of Fig. 6(a)) and all the possible modes of the oil section waveguide. Note that the computation of $T_{M}$ is performed without any approximation other than numerical discretisation [36]. In general, the selection is unambiguous since we observe that a single mode has a $T_{M}$ values much larger than the others for every value of $n_{\text {oil }}$. The transmissions $T_{t}$ and $T_{F P}$ are shown in Fig. 6(a). It is found that the radiative modes weakly impact the transmission and that they are negligible after $\sim 1 \mathrm{~mm}$ propagation distance. Knowing, that our oil droplet diameter is about $4 \mathrm{~mm}$, we infer that the FP model can be used with confidence to simulate the experimental results. 
Additionally, we observe in Fig. 6(b) that the spatial mode profile is also affected by the outer refractive index $\left(n_{\text {oil }}\right)$ of the oil droplet deposited on the glass sample. For a glass-air interface ( $n_{\text {oil }}=1$ ) as well as for $n_{\text {oil }}=1.75$, the mode is pushed back towards the inner part of the glass, as can be intuitively understood by considering the RI mismatch between the glass and upper medium. However, for $n_{\text {oil }} \sim n_{\text {glass }}=1.602$ i.e. for a well buried waveguide, the mode profile becomes centro-symmetric and well guided by the silver cluster planes, similarly to the typical type A mode reported in our previous work [28].
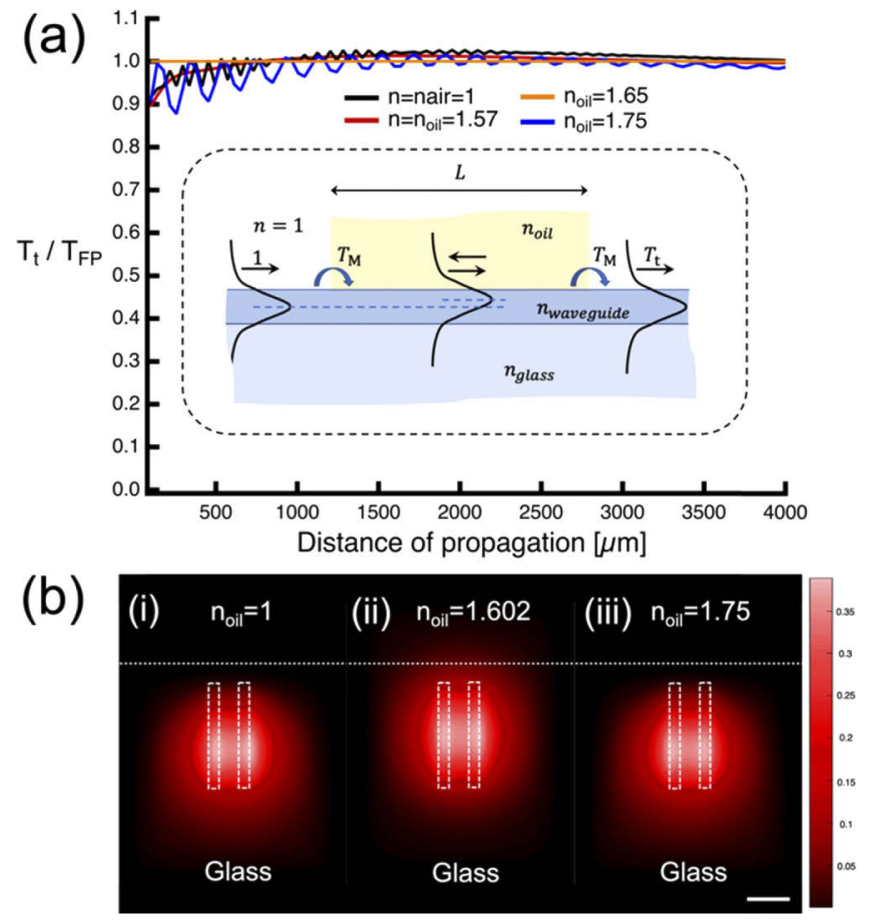

Fig. 6. (a) The normalized transmission $T_{t} / T_{F P}$ for different oil's refractive indices as a function of the propagation distance. Inset: Schematic presentation of the geometry simulated in the theoretical model. The mode propagating in the oil section is different from the mode in the air section due to refractive index mismatch. The double arrows in the oil section indicates that the numerical mode could propagate in both ways. (b) simulated near-field mode profiles for different outer refractive indexes $n$ (i) $n=1$ (air) (ii) $n=1.602$ (glass refractive index) (iii) $n=1.75$. The spatial mode profile is affected also by the outer refractive index deposited on the glass surface. The scale bare is equal to $2 \mu \mathrm{m}$.

The normalized transmission $T_{F P} / T_{1}\left(T_{1}\right.$ being the transmission for $\left.n_{o i l}=1\right)$ of the sensor is plotted as a function of the different oil's RI in Fig. 7(a) for two oil droplets length. The simulated results quantitatively predict all the main features of the experimental data. Our modeling approach is able to simulate the initial increase of the transmission for $n_{\text {oil }} \leq n_{\text {glass }}$, in contrast to the previous reported models $[5,19]$. It also predicts the sudden transmission drop for $n_{\text {oil }} \sim n_{\text {eff }}$, and a second increase for $n_{\text {oil }}>n_{\text {eff }}$ in a RI region for which all the modes of the waveguide are leaky, thereby explaining why the transmission never reaches 1 in this region. One should mention, that the previous models [5,19] do not predict the double-wing feature of the transmission for $n_{\text {oil }} \approx n_{\text {glass }}$ and thus cannot quantitatively match our experimental data.

However, we note that the numerical values of Fig. 7 (a) in the region $n_{\text {oil }}>n_{\text {eff }}$ do not exactly match the experimental data, since a better agreement is obtained for a simulated oil droplet length $L=1 \mathrm{~mm}$. The deviation could be attributed to some uncertainties concerning the $\Delta \mathrm{n}$ 
(a)

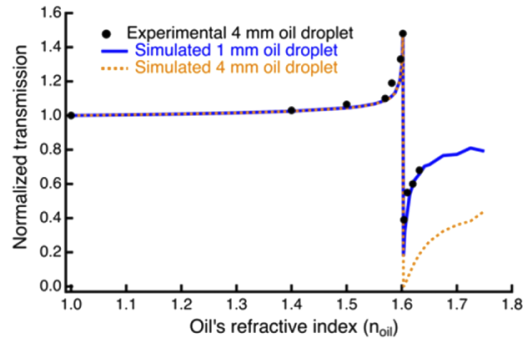

(b)

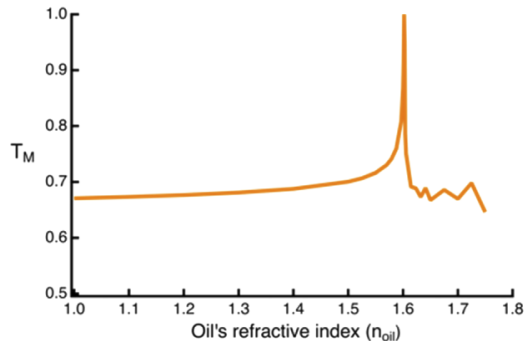

(c)

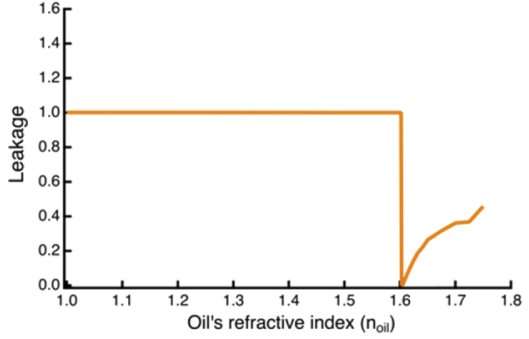

(d)

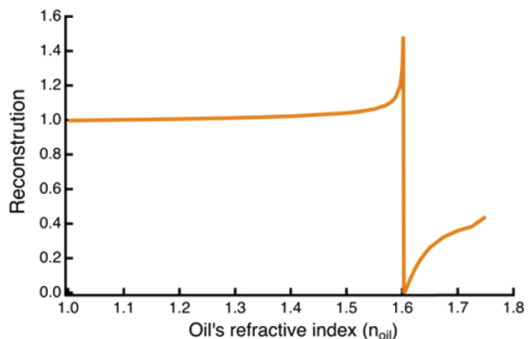

Fig. 7. (a) Simulated (blue and orange respectively for 1 and $4 \mathrm{~mm}$ oil droplet) and experimental (black dots) normalized transmission of the refractive index sensor for different oil's refractive indexes. The new theoretical methods simulate well the behavior of our refractive index sensor with the increase and decrease of the transmission. (b) The transverse mode-mismatch $T_{M}$ between the buried mode and the near-surface mode as a function of different oil RI (c) The leakage/damping of the guided mode as a function of the different RI (d) The reconstruction of the mode mismatch and leakage from Figs. 7(b) - 7(c) explaining that physical behavior of our sensor is based on the mode mismatch and mode leakage following the deposition of different oil RI.

profile distribution of the waveguide for instance or more to some effect that are not considered in our simplified model. For instance, in our model the oil thickness is assumed to be infinite, whereas it is of the order of $1 \mathrm{~mm}$ in the experiment, implying that the numerical model neglects a possible re-coupling of a portion of the leakage inside the waveguide, thereby overestimating the leakage which may explains the higher experimental transmission. Even though, one can say that the theoretical method predicts well and explains the intricate response of the RI sensor, which represents an improvement with respect to previous works $[5,19]$.

In order to have a deeper understanding of the physical mechanisms responsible for the surprising response of the waveguide sensor for $n_{o i l}<n_{\text {eff }}$, we rely on the FP model. First, we observe that the transverse-mode profile mismatch (revealed by the coupling coefficient $T_{M}$ in Fig. 7(b) between the buried waveguide mode and the near-surface waveguide mode results in a reduced transmission of $\sim 66 \%$ corresponding to transmission decrease of $\sim 34 \%$ in the region I' $\left(1<n_{\text {oil }}<n_{\text {glass }}\right)$ and in the leaky region $\left(n_{\text {oil }}>n_{\text {eff }}\right)$. When the RI of the oil equals that of the glass $\left(n_{\text {oil }}=n_{\text {glass }}\right)$, the mode mismatch vanishes, the initial losses are compensated and the modal transmission increases up to 1 (Fig. 7(b)); this explains the experimental transmission increase in Figs. 5-7(a). Then, we investigate the impact of the leakage in the leaky region by considering the imaginary part $n^{\prime \prime}$ eff of the effective index of the leaky mode. Figure 7(c) shows the damping, $\exp \left(-4 \pi n^{\prime \prime}{ }_{e f f} L / \lambda\right)$, for an oil droplet of $L=4 \mathrm{~mm}$. A strong leakage appears just above the cutoff $n_{\text {oil }}=n_{\text {glass }}$, and then progressively decreases. Finally, in Fig. 7(d), we plot the product of the transmission mismatch and the leakage damping. We infer that 1) the sensor response in region I' can be attributed to a mode mismatch issue only, 2) the double-wing response for $n_{\text {oil }} \approx n_{\text {glass }}$ results from a double effect, a rapid variation of the mismatch followed by a sharp enhancement 
of the leakage, and 3$)$ the transmission in region III $\left(n_{\text {oil }}>n_{\text {eff }}\right)$ is mainly impacted by a decrease of the leakage of the dominant mode.

\section{Conclusion}

Taking advantage of the low pulse energy inscription regime of type Argentum compared to type I, laser writing on the glass surface is demonstrated with no need for additional processing. For sensing applications, near-surface waveguides were written in silver containing glass. No etching, nor reinforced glass nor compensation methods are required to inscribe those waveguides compatible with sensing applications. Based on this approach, a refractive index (RI) sensor was created in a $1 \mathrm{~cm}$ glass chip that shows a double-wing feature that sharpens the response shape and enhance the sensitivity. An increase of $\sim 50 \%$ in the transmission followed by a decrease of $\sim 66 \%$ were observed for a $4 \mathrm{~mm}$-long oil droplet ( $n_{\text {oil }}=1.602$ and 1.604 respectively). These surface waveguides remarkably show a non-null sensitivity for oil indices below the glass index, even for indices down to the 1.33-1.37 range compatible with biological and environmental applications. For waveguides that are embedded deeper inside the bulk, only a single wing feature is observed which is the transmission drop corresponding to the oil's RI matching the waveguide's RI. Moreover, a new theoretical modeling approach is proposed based on the solution of the vectorial Maxwell equations of the guided modes propagating in near-surface waveguides. This approach is the first one to predict and explain the increase in the transmission for which the oil's RI is lower than the waveguide's $\left(n_{\text {oil }}<n_{\text {eff }}\right)$. Finally, a sensitive RI sensor operating in the range of $1 \rightarrow 1.75$ refractive index exhibiting transmission drop up to $80 \%(7 \mathrm{~dB})$ for an interaction length of $5 \mathrm{~mm}$ is manufactured in a $1 \mathrm{~cm}$ silver containing glass chip which is a first using fs LW technique. Those results pave the wave towards sensing applications based on type Argentum waveguides in silver containing glasses.

\section{Funding}

Agence Nationale de la Recherche (10-IDEX-03-02); Natural Sciences and Engineering Research Council of Canada (CG101779); Canada Foundation for Innovation (GF072345); Fonds de Recherche du Québec - Nature et Technologies (CO201310, FT097991); H2020 Marie Skłodowska-Curie Actions (823941).

\section{Acknowledgments}

Authors would like to thank Sylvain Danto and Thierry Cardinal for providing homemade glasses for primary tests, Jérôme Lapointe for the fruitful discussions, Stéphane Gagnon for cutting and polishing of the glass samples and Denis Boudreau for lending the lab equipment. PL thank Jean Paul Hugonin for a longstanding, fruitful and friendly collaboration.

\section{Disclosures}

The authors declare that there are no conflicts of interest related to this article.

\section{References}

1. H. Tai, H. Tanaka, and T. Yoshino, "Fiber-optic evanescent-wave methane-gas sensor using optical absorption for the 3.392- $\mu$ m line of a He-Ne laser," Opt. Lett. 12(6), 437-439 (1987).

2. J. Heo, M. Rodrigues, S. J. Saggese, and G. H. Sigel, "Remote fiber-optic chemical sensing using evanescent-wave interactions in chalcogenide glass fibers," Appl. Opt. 30(27), 3944-3951 (1991).

3. P. S. Kumar, C. P. G. Vallabhan, V. P. N. Nampoori, V. N. S. Pillai, and P. Radhakrishnan, "A fibre optic evanescent wave sensor used for the detection of trace nitrites in water," J. Opt. A: Pure Appl. Opt. 4(3), 247-250 (2002).

4. P. Polynkin, A. Polynkin, N. Peyghambarian, and M. Mansuripur, "Evanescent field-based optical fiber sensing device for measuring the refractive index of liquids in microfluidic channels," Opt. Lett. 30(11), 1273-1275 (2005).

5. L. Bilro, N. Alberto, J. L. Pinto, and R. N. Nogueira, "A simple and low-cost cure monitoring system based on a side-polished plastic optical fibre,” Meas. Sci. Technol. 21(11), 117001 (2010). 
6. R. M. Ribeiro, J. L. P. Canedo, M. M. Werneck, and L. R. Kawase, "An evanescent-coupling plastic optical fibre refractometer and absorptionmeter based on surface light scattering," Sens. Actuators, A 101(1-2), 69-76 (2002).

7. J. H. Chong, P. Shum, H. Haryono, A. Yohana, M. K. Rao, C. Lu, and Y. Zhu, "Measurements of refractive index sensitivity using long-period grating refractometer," Opt. Commun. 229(1-6), 65-69 (2004).

8. A. N. Chryssis, S. M. Lee, S. B. Lee, S. S. Saini, and M. Dagenais, "High sensitivity evanescent field fiber Bragg grating sensor," IEEE Photonics Technol. Lett. 17(6), 1253-1255 (2005).

9. C. Lin, C. Liao, J. Wang, J. He, Y. Wang, Z. Li, T. Yang, F. Zhu, K. Yang, Z. Zhang, and Y. Wang, "Fiber surface Bragg grating waveguide for refractive index measurements," Opt. Lett. 42(9), 1684-1687 (2017).

10. J. Bures and R. Ghosh, "Power density of the evanescent field in the vicinity of a tapered fiber," J. Opt. Soc. Am. A 16(8), 1992-1996 (1999).

11. R. M. Vazquez, R. Osellame, D. Nolli, C. Dongre, H. van den Vlekkert, R. Ramponi, M. Pollnau, and G. Cerullo, "Integration of femtosecond laser written optical waveguides in a lab-on-chip," Lab Chip 9(1), 91-96 (2009).

12. G. Cerullo, R. Osellame, S. Taccheo, M. Marangoni, D. Polli, R. Ramponi, P. Laporta, and S. De Silvestri, "Femtosecond micromachining of symmetric waveguides at $1.5 \mu \mathrm{m}$ by astigmatic beam focusing," Opt. Lett. 27(21), 1938-1940 (2002).

13. S. M. Eaton, M. L. Ng, R. Osellame, and P. R. Herman, "High refractive index contrast in fused silica waveguides by tightly focused, high-repetition rate femtosecond laser," J. Non-Cryst. Solids 357(11-13), 2387-2391 (2011).

14. D. G. Lancaster, S. Gross, H. Ebendorff-Heidepriem, K. Kuan, T. M. Monro, M. Ams, A. Fuerbach, and M. J. Withford, "Fifty percent internal slope efficiency femtosecond direct-written Tm3+: ZBLAN waveguide laser," Opt. Lett. 36(9), 1587-1589 (2011).

15. L. B. Fletcher, J. J. Witcher, N. Troy, S. T. Reis, R. K. Brow, and D. M. Krol, "Direct femtosecond laser waveguide writing inside zinc phosphate glass," Opt. Express 19(9), 7929-7936 (2011).

16. J.-P. Bérubé, M. Bernier, and R. Vallée, "Femtosecond laser-induced refractive index modifications in fluoride glass," Opt. Mater. Express 3(5), 598-611 (2013).

17. J. Lapointe, M. Gagne, M. J. Li, and R. Kashyap, "Making smart phones smarter with photonics," Opt. Express 22(13), 15473-15483 (2014).

18. G. Della Valle, R. Osellame, and P. Laporta, "Micromachining of photonic devices by femtosecond laser pulses," J. Opt. A: Pure Appl. Opt. 11(1), 013001 (2009).

19. J. Lapointe, F. Parent, E. S. de Lima Filho, S. Loranger, and R. Kashyap, "Toward the integration of optical sensors in smartphone screens using femtosecond laser writing," Opt. Lett. 40(23), 5654-5657 (2015).

20. J.-P. Bérubé and R. Vallée, "Femtosecond laser direct inscription of surface skimming waveguides in bulk glass," Opt. Lett. 41(13), 3074-3077 (2016).

21. J. Martínez, A. Ródenas, A. Stake, M. Traveria, M. Aguiló, J. Solis, R. Osellame, T. Tanaka, B. Berton, S. Kimura, N. Rehfeld, and F. Díaz, "Harsh-Environment-Resistant OH-Vibrations-Sensitive Mid-Infrared Water-Ice Photonic Sensor," Adv. Mater. Technol. 2(8), 1700085 (2017).

22. E. Zgraggen, O. Scholder, G. L. Bona, F. Fontana, E. Alberti, A. Crespi, R. Osellame, T. Scharf, and I. Shorubalko, "Optical properties of waveguide-coupled nanowires for sub-wavelength detection in microspectrometer applications," J. Opt. 17(2), 025801 (2015).

23. V. Maselli, J. R. Grenier, S. Ho, and P. R. Herman, "Femtosecond laser written optofluidic sensor: Bragg grating waveguide evanescent probing of microfluidic channel," Opt. Express 17(14), 11719-11729 (2009).

24. K. Zhou, Y. Lai, X. Chen, K. Sugden, L. Zhang, and I. Bennion, "A refractometer based on a micro-slot in a fiber Bragg grating formed by chemically assisted femtosecond laser processing," Opt. Express 15(24), 15848-15853 (2007).

25. H. O. Çirkinoğlu, M. M. Bayer, U. S. Gökay, A. Serpengüzel, B. Sotillo, V. Bharadwaj, R. Ramponi, and S. M. Eaton, "Silicon microsphere whispering gallery modes excited by femtosecond-laser-inscribed glass waveguides," Appl. Opt. 57(14), 3687-3692 (2018).

26. D. Liu, Y. Li, R. An, Y. Dou, H. Yang, and Q. Gong, "Influence of focusing depth on the microfabrication of waveguides inside silica glass by femtosecond laser direct writing," Appl. Phys. A 84(3), 257-260 (2006).

27. A. Abou Khalil, J.-P. Bérubé, S. Danto, T. Cardinal, Y. Petit, L. Canioni, and R. Vallée, "Comparative study between the standard type I and the type A femtosecond laser induced refractive index change in silver containing glasses," Opt. Mater. Express 9(6), 2640 (2019).

28. A. Abou Khalil, J. P. Berube, S. Danto, J. C. Desmoulin, T. Cardinal, Y. Petit, R. Vallee, and L. Canioni, "Direct laser writing of a new type of waveguides in silver containing glasses," Sci. Rep. 7(1), 11124 (2017).

29. Y. Petit, S. Danto, T. Guérineau, A. Abou Khalil, A. Le Camus, E. Fargin, G. Duchateau, J.-P. Bérubé, R. Vallée, Y. Messaddeq, T. Cardinal, and L. Canioni, "On the femtosecond laser-induced photochemistry in silver-containing oxide glasses: mechanisms, related optical and physico-chemical properties, and technological applications," Adv. Opt. Technol. 7(5), 291-309 (2018).

30. M. Bellec, A. Royon, B. Bousquet, K. Bourhis, M. Treguer, T. Cardinal, M. Richardson, and L. Canioni, "Beat the diffraction limit in 3D direct laser writing in photosensitive glass," Opt. Express 17(12), 10304-10318 (2009).

31. M. Bellec, A. Royon, K. Bourhis, J. Choi, B. Bousquet, M. Treguer, T. Cardinal, J.-J. Videau, M. Richardson, and L. Canioni, "3D Patterning at the Nanoscale of Fluorescent Emitters in Glass," J. Phys. Chem. C 114(37), 15584-15588 (2010). 


\section{Optics EXPRESS}

32. J.-C. Desmoulin, Y. Petit, L. Canioni, M. Dussauze, M. Lahaye, H. M. Gonzalez, E. Brasselet, and T. Cardinal, "Femtosecond laser structuring of silver-containing glass: Silver redistribution, selective etching, and surface topology engineering," J. Appl. Phys. 118(21), 213104 (2015).

33. K. Bourhis, A. Royon, M. Bellec, J. Choi, A. Fargues, M. Treguer, J.-J. Videau, D. Talaga, M. Richardson, T. Cardinal, and L. Canioni, "Femtosecond laser structuring and optical properties of a silver and zinc phosphate glass," J. Non-Cryst. Solids 356(44-49), 2658-2665 (2010).

34. E. Smetanina, B. Chimier, Y. Petit, N. Varkentina, E. Fargin, L. Hirsch, T. Cardinal, L. Canioni, and G. Duchateau, "Modeling of cluster organization in metal-doped oxide glasses irradiated by a train of femtosecond laser pulses," Phys. Rev. A 93(1), 013846 (2016).

35. M. J. F. Digonnet, J. R. Feth, L. F. Stokes, and H. J. Shaw, "Measurement of the core proximity in polished fiber substrates and couplers," Opt. Lett. 10(9), 463-465 (1985).

36. E. Silberstein, P. Lalanne, J.-P. Hugonin, and Q. Cao, "Use of grating theories in integrated optics," J. Opt. Soc. Am. A 18(11), 2865-2875 (2001).

37. P. Lalanne, "Electromagnetic analysis of photonic crystal waveguides operating above the light cone," IEEE J. Quantum Electron. 38(7), 800-804 (2002).

38. P. Lalanne, M. Besbes, J. P. Hugonin, S. van Haver, O. T. A. Janssen, A. M. Nugrowati, M. Xu, S. F. Pereira, H. Urbach, A. S. van de Nes, P. Bienstman, G. Granet, A. Moreau, S. Helfert, M. Sukharev, T. Seideman, F. Baida, B. Guizal, and D. van Labeke, "Numerical analysis of a slit-groove diffraction problem," JEOS:RP 2, 07022 (2007). 